\title{
Breathe: psychological comorbidity of respiratory illness
}

Welcome to the March issue of Breathe!

This issue is dedicated to psychological aspects of respiratory illness, including two papers dedicated to functional disorders presenting as respiratory morbidity: wheeze and cough. Though with a paediatric focus, the investigation and management remain broadly similar to that in adults.

In this issue, we also have an excellent guide on how to distinguish dysfunctional breathing from impaired breathing due to pulmonary or muscular pathology; useful physiological tests that play a role in improving our management of patients with sometimes very worrying symptoms.

We have some excellent online exclusives which will appear in due course, so please, ensure that you return to the Breathe website to read and share this material (breathe.ersjournals.com).

Frits Franssen has provided an editorial on the inaugural online journal club, which took place in December 2016. The speaker, panellists and attendees all agreed that it was an enjoyable and educational session, so I would like to encourage all of you who can make the time to take part. The next journal club will be held on March 7, 2017, with the general theme related to mental health issues in COPD and whether they impact on longevity. We hope that the journal club sessions will be accredited for continuing medical education and the team at Breathe are working on this.

Additionally, we have an editorial on the upcoming Sleep and Breathing Conference in Marseille, France, in April. Those of you who have an interest in respiratory sleep are encouraged to attend this excellent conference, which is largely education focussed. We are piloting the new HERMES sleep programme in several of the workshops this year, so your input would be much valued.

Again, I thank all contributors to Breathe and the editorial team. Please contribute your case reports, your reviews, your editorials and your comments; we welcome them all.

With the longer days of Spring, may your work, education and research continue to flourish. Happy reading!

\section{Conflict of interest}

None declared.

Cite as: Riha RL. Breathe: psychological comorbidity of respiratory illness. Breathe 2017; 13: 3. 